\title{
El sector cerámico español: Una oportunidad de negocio para empresas de servicios de consultoría ERP
}

\author{
R. F. OLTRA-BADENES ${ }^{1}$ Y H. GIL-GÓMEZ
}

'Dpto. de Organización de Empresas. Universitat Politècnica de València. Valencia, España

\begin{abstract}
El sector cerámico es un sector estratégico en España, que está perdiendo cuota de mercado ante la producción de países emergentes. Para conseguir ventaja competitiva frente a otros países que compiten en coste, una de las oportunidades de mejora del sector cerámico en España, es incrementar y optimizar el empleo de las TIC. Sin embargo, actualmente no hay sistemas desarrollados que den solución adecuada a los problemas específicos del sector cerámico.

Por tanto, surge una oportunidad de negocio para empresas que ofrecen servicios de consultoría de implantación de sistemas de información, como son los Enterprise Resource Planning (ERP). Para ello, en primer lugar es necesario conocer el sector, y sus problemáticas concretas, con el objetivo de desarrollar un ERP adecuado. Este artículo identifica dicha oportunidad de negocio y presenta una de las problemáticas más específicas del sector, que no está implementada en ningún sistema ERP, con el objetivo de que las empresas de servicios de consultoría ERP la conozcan y sean capaces de desarrollar una solución adecuada.
\end{abstract}

Palabras clave: ERP, solución sectorial, solución vertical, sector cerámico, falta de homogeneidad en el producto.

The Spanish ceramic industry: a business opportunity for ERP consultant services enterprises

The ceramic industry is a strategic sector in Spain, which is losing market share to the production of emerging countries. To gain competitive advantage over other countries competing in cost, one of the opportunities for improvement of the ceramic industry in Spain , is to increase and optimize the use of ICT. However, there is currently no developed systems that provide appropriate to the specific problems of the ceramic sector solution.

Therefore, a business opportunity arises for companies that provide consulting services for the implementation of information systems, such as Enterprise Resource Planning System (ERP). To do this, first we need to know the industry and its specific problems, with the goal of developing a suitable ERP. This paper identifies such business opportunity and presents one of the more specific problems of the sector, which is not implemented in any ERP system. Then, ERP consulting services companies will have the knowledge of the problem and they will be able to develop an appropriate solution.

Keywords: ERP, industry solution, vertical solution, ceramic industry, lack of de homogeneity in the product.

\section{INTRODUCCIÓN}

El sector cerámico es uno de los sectores productivos más importantes en España, siendo además uno de los principales productores a nivel mundial (1). Sin embargo, en los últimos años, su peso específico en el mercado internacional ha disminuido, y la tendencia es que siga haciéndolo en beneficio de países emergentes, que se están consolidando rápidamente (2). Los productores de baldosa cerámica españoles no pueden competir en coste, y deben hacerlo mediante otras estrategias, como puede ser la diferenciación, calidad y diseño del producto o la productividad (3). Para ello, es necesario un uso adecuado de las TIC. Sin embargo, en este momento el uso de las TIC, y en concreto de los sistemas de información (SI) para la gestión de empresas, no está suficientemente desarrollado (4).

Para aprovechar las TIC de forma adecuada, uno de los problemas ante los que se encuentra éste sector, es que no hay SI adaptados a su problemática particular. En consecuencia, si una empresa es capaz de ofrecer un SI adaptado de forma específica al sector cerámico español, tendrá una oportunidad de negocio.
El objetivo de este artículo es identificar esta oportunidad de negocio, para que emprendedores, o empresas de servicios TIC ya existentes puedan aprovecharla, pudiendo acceder a un mercado importante y estratégico en España. Además, ese mercado se encuentra muy localizado en una zona geográfica reducida y muy concreta, ya que el $83 \%$ de las empresas azulejeras españolas están situadas en la Comunidad Valenciana, prácticamente todas en la provincia de Castellón (5). Esta característica hace que se puedan concentrar las acciones y esfuerzos emprendedores para satisfacer esta necesidad del sector cerámico.

\section{EL SECTOR CERÁMICO EN ESPAÑA.}

La producción mundial de cerámica se concentra en pocos países. España e Italia son los principales países productores en Europa, siendo España el segundo país exportador europeo 
y tercero mundial. De esta forma, el sector cerámico es estratégico en la economía española, con un $65 \%$ de su facturación correspondiente a exportaciones, que lo sitúan entre los doce mayores exportadores de España y como la segunda industria que mayor superávit aporta a la balanza comercial del país, pese a que el entramado cerámico español está compuesto en su práctica totalidad por pymes y empresas de origen familiar (1)

Sin embargo, en los últimos años se está produciendo una pérdida de cuota de mercado frente a países como Vietnam o Irán que, basándose en ventajas como los inferiores costes de mano de obra y materias primas, se están consolidando de una manera rápida (2). También en (3) se cita la amenaza que los países de la Europa del Este suponen para el sector azulejero español e italiano, ya que tienen acceso a la tecnología necesaria para fabricar azulejos, con el condicionante de que la mano de obra en esos países, es mucho más barata.

Ante esta situación el reto que se les plantea a los países que tradicionalmente han liderado el mercado, como España o Italia, es seguir una clara estrategia de diferenciación basada en el diseño, la calidad y el uso eficiente de las TICS (2), (3).

\section{EL SECTOR CERÁMICO Y LAS TIC}

La utilización de las TIC en el sector cerámico español es escasa. A pesar de que este sector ha incorporado tecnología de alto nivel en las fases productivas y especialmente en la preparación de la materia prima y decoración, no ha ocurrido así con las metodologías y los procesos de gestión (2), (3). Centrando la atención en los SI, se puede afirmar que actualmente, dentro del sector cerámico de la Comunidad Valenciana, el tipo de software utilizado se limita en la mayoría de los casos al uso de hojas de cálculo $(100 \%)$ y bases de datos $(80 \%)$ (3). Evidentemente, este tipo de software no es capaz de gestionar los procesos de la empresa cerámica, al menos de forma eficaz.

No obstante, las medianas y grandes empresas utilizan también software a medida o sistemas ERP, destacando en este caso que las empresas líderes en el sector están incorporando, o han incorporado recientemente, sistemas ERP. Esta tendencia será seguida en breve por el resto de empresas del sector (3). De esta forma, en los próximos años, los ERP se introducirán progresivamente en el sector cerámico de la Comunidad Valenciana.

Los SI que emplean actualmente las empresas cerámicas ofrecen algunas ventajas, pero también inconvenientes, algunos de ellos importantes y que es necesario mejorar. Como ventajas que ofrecen estos sistemas, se puede destacar el fácil uso, la flexibilidad y la integración en la empresa. Pero es muy importante destacar que entre las desventajas más frecuentes, se encuentran que no se satisfacen los requerimientos específicos del sector cerámico, destacando que no hay herramientas que permitan resolver adecuadamente los problemas actuales de diversificación y diferenciación de productos (4), lo cual es básico para una gestión de operaciones adecuada y por tanto para la satisfacción del cliente, algo imprescindible en un entorno tan competitivo como el actual.

\section{NECESIDAD DE SERVICIOS DE CONSULTORÍA Y DESARROLLO DE SOFTWARE ESPECÍFICO PARA EL SECTOR CERÁMICO. ESTRATEGIA DE ENTRADA EN EL MERCADO}

Tras la exposición realizada en el apartado anterior, parece evidente que, para posibilitar una gestión eficaz y eficiente en las empresas cerámicas, es necesario un SI adecuado a los requerimientos específicos del sector cerámico. Además, la situación actual de este sector, indica que la demanda del SI adaptado al sector sería alta, ya que es un factor estratégico que debe mejorar si no quiere continuar perdiendo cuota de mercado.

Por tanto, surge una importante oportunidad de negocio. Si una empresa de servicios de consultoría, conocedora de la problemática concreta del sector cerámico, es capaz de desarrollar un SI adaptado a los requisitos que dicha problemática genera, tendrá una clara oportunidad de negocio. Para ello, debe adoptar una "estrategia vertical".

Una estrategia vertical está dirigida a minimizar el "gap" existente entre los procesos de negocio de un sector y los procesos del SI. De este modo, los proveedores de SI que emplean una estrategia vertical, desarrollan versiones especializadas de los SI para mercados verticales.

Sin embargo, las empresas de servicios de consultoría de SI, que quieran entrar a competir en el mercado, pueden poner en marcha esta estrategia en más de una forma (6). Una opción es comenzar el desarrollo de productos a partir de cero. Sin embargo, en este caso, el proceso de $\mathrm{I}+\mathrm{D}+\mathrm{i}$ de estos productos es largo y complejo, y requiere grandes inversiones.

Otra opción es colaborar con un proveedor dominante existente, por lo general un proveedor de SI de gran tamaño, y aprovechar el conocimiento y presencia en el mercado, adaptando el SI genérico que ofrece, a los requerimientos específicos del sector que se va a "verticalizar". Así, un nuevo participante puede tomar ventaja de las plataformas ya desarrolladas y funcionalidades, como base para su producto, y desarrollar soluciones para las necesidades y requisitos específicos de su objetivo vertical.

Consideramos que esta estrategia es la adecuada en este caso, en la que se tomaría un SI genérico en el mercado, y se adaptaría al sector cerámico, desarrollando los requerimientos específicos del cerámico sobre la base del SI existente.

En la actualidad, la solución de referencia para SI de las empresas en todo el mundo son los ERP (7). Por tanto, una forma de poner en marcha la estrategia vertical de una empresa de servicios de consultoría de SI, para atacar a un sector concreto, puede ser la de seleccionar un ERP existente en el mercado, y adaptarlo al sector en concreto. Este tipo de sistemas ERP particularizados a las necesidades de un sector en concreto, se denominan ERP vertical o ERP sectorial. A continuación, en el apartado siguiente, se explica el significado de este concepto.

\section{LOS ERP VERTICALES O SECTORIALES}

LosERPson software para la gestión deempresa, concebidos y desarrollados como sistemas de gestión genéricos, aplicables a los diferentes procesos de cualquier empresa. (6). Este es el concepto que se denomina en el ámbito del software para empresas como "solución horizontal", en la que el sistema 
proporciona una funcionalidad estándar, que se supone capaz de soportar los procesos de cualquier organización.

Sin embargo, diferentes organizaciones, que además pueden pertenecer a diferentes sectores industriales, tienen procesos de negocio diferentes, y debido ello, también tienen requerimientos diferentes en cuanto a los sistemas de gestión. Por supuesto, esto es conocido por los fabricantes de ERP's, y por ello, desarrollan sus sistemas de forma que permitan cierta personalización o "customización" a través de una estructura modular y de su parametrización y configuración. (6).

De esta forma, los sistemas ERP se componen de "módulos", que las empresas contratan o activan en función de sus necesidades. Por ejemplo, una empresa dedicada a la exportación de productos cítricos, no necesita el módulo de producción, o una empresa de prestación de servicios de consultoría, no necesita el módulo de gestión de stocks. Esta característica modular de los sistemas ERP es un mecanismo que facilita su implantación (8).

Además, en cada uno de sus módulos, los sistemas ERP tienen diferentes opciones de funcionamiento, que el usuario debe seleccionar para que el sistema trabaje y ejecute los procesos de una u otra forma determinada. Esto es lo que se llama "configuración y parametrización del sistema".

Pero pese a estas opciones de ajustar el sistema a las necesidades de cada empresa, la idea de sistemas genéricos con una filosofía horizontal o "one to fit all" ("uno que sirva para todos") (6), con la capacidad de acoplarse con éxito y servir para cualquier organización, presenta algunos problemas en el momento de aplicarse y cubrir las necesidades de las organizaciones (9).

Estos problemas se presentan básicamente en algunas áreas de la empresa, como pueden ser la de logística, producción, gestión de stock o ventas, en las que puede haber muchas diferencias entre las necesidades de diferentes empresas, y sobre todo cuando estas pertenecen a diferentes sectores industriales.

En un sistema "one to fit all", estas particularidades necesarias para un sector en concreto no tienen cabida, y deben ser desarrolladas de forma adicional al ERP, en cada implantación y para cada empresa particular, como módulos adicionales o modificaciones de los módulos que componen el sistema. Ello hace que el proceso de implantación del ERP sea más complejo, largo y costoso, debido a que es necesario dedicar recursos al desarrollo de modificaciones y módulos adicionales, además del proceso de implantación que ya de por sí es complejo, costoso y con riesgos (10).

Por ello, en los últimos años, los proveedores de software de gestión han asumido el reto de adaptar mejor los productos al funcionamiento real de las empresas, y un número cada vez mayor de ellos ha adoptado una estrategia de segmentación, es decir, la "verticalización" o lo que se denomina soluciones "verticales" o "sectoriales", en lo que parece ser uno de las más evidentes tendencias estratégicas en los mercados de software de empresa (11).

Esta estrategia, la "verticalización" se puede definir como la personalización de las aplicaciones dentro de industrias específicas (12). De esta forma, una solución vertical desarrollada para un sector concreto, incluirá todas aquellas características diferenciales que dicho sector necesita para su gestión de la información. Con ello, las empresas de ese sector que implanten la solución no necesitarán modificar el sistema para adaptarlo a sus necesidades básicas, dado que estas necesidades ya estarán incluidas en la solución vertical o sectorial, y la implantación será mucho menos costosa y con muchas más probabilidades de éxito.

Por supuesto, el primer paso hacia la verticalización es incorporar los conocimientos específicos del sector (12), por lo que los desarrolladores del software deben conocer perfectamente su problemática. Para ello, en muchas ocasiones se utilizan empresas características del sector, que pueden actuar como empresas para "implantaciones piloto", que permitan a los desarrolladores adquirir el conocimiento necesario, no solo teórico sino también práctico, y probar la solución vertical en un entorno real.

Existen trabajos que estudian las soluciones ERP verticales existentes en todo el mundo (13), (14) de los cuales se puede concluir que actualmente no existen soluciones verticales que cubran los requerimientos específicos del sector cerámico.

Por tanto, ante una necesidad existente en el mercado, parece que la opción de desarrollar una solución ERP vertical, que sea capaz de gestionar la problemática característica del sector cerámico, puede ser una oportunidad de negocio muy interesante.

Con ese objetivo, a continuación se analiza uno de los requerimientos funcionales que un sistema ERP vertical para el sector cerámico debe cumplir, como es la correcta gestión del producto, incluyendo las características de tono y calibre.

\section{REQUERIMIENTO FUNCIONAL PARA UN ERP VERTICAL ADAPTADO AL SECTOR CERÁMICO. LA PROBLEMÁTICA DEL TONO Y CALIBRE EN EL SECTOR CERÁMICO}

La producción de azulejos puede verse afectada por factores externos, tales como la humedad, la temperatura, el origen de las arcillas y pigmentos. Las variaciones en estos factores incontrolados provocan pequeñas diferencias en el color y la textura de los azulejos (15). Por este motivo, "aparte de posibles defectos, el proceso de producción, y sobre todo los ciclos de producción cortos, generan falta de uniformidad en los colores (diversidad de tonos) y en los tamaños (diversidad de calibres)"(16). Sin embargo, a pesar de que en un mismo producto existan diferencias de tonos y calibres, es importante que al aplicar los azulejos (en un revestimiento o pavimento), en cada juego de azulejos, cada azulejo sea de aspecto similar (mismo tono y calibre) (17). Esta característica de la empresa cerámica genera diferentes problemáticas en la gestión de operaciones, que la hacen más compleja de lo habitual (16).

Esta circunstancia fuerza la necesidad de clasificar toda la producción en función de los valores de estas variables. Por ello es necesaria la fase de clasificación (4) en la cual, los productos se clasifican y agrupan en función de su tono y calibre, para su posterior embalado y paletizado. A partir de ese momento, los productos deben quedar perfectamente identificados, y dispuestos en el almacén con su tono y calibre perfectamente definidos, de manera que puedan gestionarse dichos productos en base a estos valores. Por ello, es muy importante una clasificación correcta y adecuada de la fabricación.

Sin embargo, para ello, es fundamental e imprescindible que exista un SI adecuado, que sea capaz de recoger y gestionar la información necesaria para el manejo de las diversos tonos y calibres de cada producto. Este sistema además, deberá ser capaz de gestionar y dar soporte a la toma de decisiones en los diferentes procesos de la empresa, como son la gestión de inventario, la 
gestión de pedidos, la planificación y gestión de la producción o la previsión de la demanda. Evidentemente, el SI, para la gestión de estos procesos, debe tener en cuenta la clasificación de los productos y sus valores en cuanto a tono y calibre. Precisamente, este es uno de los problemas que no resuelven actualmente los SI actuales, la diversificación de productos en función del tono y calibre, y la identificación correcta de ellos.

$\mathrm{Si}$ el producto no está perfectamente identificado, con su tono y calibre perfectamente definidos, la gestión de pedidos será más compleja, debido a que será más complejo localizar en el almacén los productos que puedan satisfacer las necesidades de los clientes. Esta es una situación que incide directamente en el servicio al cliente, y que puede ser una fuente de errores, de devoluciones de pedidos, insatisfacción del cliente, pérdida de pedidos, de imagen de marca e incluso pérdida de clientes.

Cabe indicar que para las empresas españolas, uno de los aspectos más críticos es el cumplimiento de los plazos de entrega, y para ello, es imprescindible un buen SI, que pueda suministrar y gestionar los datos adecuados de los productos y la situación del inventario, incluyendo sus características particulares en cuanto a tono y calibre.

A estas circunstancias se añade que el clúster cerámico español muestra un enfoque hacia el cliente débil y ciertas deficiencias en la gestión de las fases finales de la cadena de valor (Distribución, Servicios Post-venta, Colocación, etc.)(3). En este sentido, la literatura especializada reconoce el uso intensivo de las TIC como elementos estratégico para una orientación proactiva hacia el cliente. Y por supuesto, para un buen servicio al cliente y su satisfacción, se hace necesaria una gestión adecuada del producto que se le sirve y por tanto, un SI que sea capaz de dar soporte a dicha gestión.

Por todo ello, un SI adaptado al sistema cerámico, un ERP vertical, que tenga en consideración estas problemática concreta, puede aportar grandes beneficios a las empresas que lo utilicen, y diferenciarlos de la competencia en respuesta y calidad de servicio al cliente, lo que redundará en una mayor satisfacción y de éste.

\section{CONCLUSIONES}

Una de las oportunidades de mejora en el sector cerámico español, y en consecuencia, una oportunidad de luchar con la pérdida de mercado existente en dicho sector, es la de potenciar el uso de las TIC, y utilizar sistemas del tipo ERP, para optimizar sus procesos y obtener ventajas competitivas.

Sin embargo, no existen sistemas ERP adaptados a las necesidades específicas del sector cerámico, por lo que existe una oportunidad de negocio para empresas que puedan ofrecer a este sector un sistema ERP adaptado a sus necesidades particulares, un ERP vertical para el sector cerámico. Evidentemente, dado que dicho ERP vertical no existe, es necesario desarrollarlo. Por ello, en este artículo se identifica adicionalmente un ejemplo de un requerimiento concreto y específico del sector cerámico, como es la problemática que genera la FHP. Este ejemplo sirve de base de partida para desarrollar la adaptación de un ERP a uno de los requerimientos más característicos del sector cerámico, que no tiene solución en los ERP actuales, como es la gestión de la problemática del tono y calibre en el producto cerámico.

Tras detectar esta oportunidad de negocio en el mercado cerámico español, surgen líneas de investigación futuras en dos direcciones básicas:
Por una parte, la investigación desde un punto de vista empresarial, desde el que se debe estudiar cómo realizar la entrada en el mercado de la nueva empresa de servicios de consultoría ERP. Por ejemplo, una colaboración con una empresa líder del sector cerámico, en la que se pueda hacer una implantación piloto del ERP vertical desarrollado, y que además colabore en el desarrollo desde un punto de vista de usuario final. O quizá a través de asociaciones de empresas del sector, como puede ser la Asociación Española de Fabricantes de Azulejos y Pavimentos Cerámicos (ASCER), que puedan promover el ERP vertical entre sus asociados. O estableciendo otro tipo de alianzas, por ejemplo con empresas de consultoría (ya sean TIC o a nivel de negocio) ya presentes en el sector.

Por otra parte, desde un punto de vista más técnico, hay que desarrollar la solución ERP vertical completa, incluyendo tanto el desarrollo completo del requerimiento identificado en este artículo, como el resto de los requerimientos específicos del sector cerámico. Por ejemplo, los requerimientos que genera la planificación de producción en base a las capacidades de los hornos de cocción de baldosa cerámica.

\section{BIBLIOGRAFÍA}

1. Asociación Española de Fabricantes de Azulejos y Pavimentos Cerámicos, «ASCER». Disponible en: http://www.ascer.es/. (Accedido: 31-mar2014).

2. J. Albors, P. Márquez, y M. Segarra, «Internet como herramienta de creación de valor en sectores maduros. El caso de los productores y distribuidores cerámicos en España», Bol.Soc.Esp.Ceram.Vidrio, vol. 48, nº. 6. 279-288, (2009).

3. J. Albors y J. L. Hervas, «La Industria cerámica europea en el siglo XXI. Retos tecnológicos y desafíos de la próxima década», Bol.Soc.Esp.Ceram. Vidrio, vol. 45, n⿳.1. 13-21, (2006).

4. R. F. Oltra-Badenes, H. Gil-Gómez, S. Asensio-Cuesta, y R. Bellver-López, «Análisis de requerimientos y diseño de modelo de datos para el desarrollo de una solución ERP vertical adaptada al sector cerámico», Bol.Soc.Esp. Ceram.Vidrio,vol. 48, n. ${ }^{\circ}$ 11. 63-70, (2013).

5. E. Vallada, C. Maroto, R. Ruiz, y B. Segura, «Análisis de la programación de la producción en el sector cerámico español», Bol.Soc.Esp.Ceram.Vidrio, vol. 44, n. ${ }^{\circ}$ 1.39-44, (2005).

6. L. Fink y S. Markovich, «Generic verticalization strategies in enterprise system markets: An exploratory framework», J.Inform.Tech., vol. 23. 281296, (2008).

7. B. Grabot y V. Botta-Genoulaz, «Special issue on Enterprise Resource Planning (ERP) systems», Comput.Ind., vol. 56, nº. 6. 507-509, (2005).

8. R. F. Oltra-Badenes, «Identificación de tendencias de los Sistemas Integrados de Gestión Empresarial. Análisis funcional y diseño de herramientas para el desarrollo de un ERP vertical adaptado al sector cerámico», Universitat Politècnica de València, Valencia, 2012.

9. T. F. Gattiker y D. L. Goodhue, «Understanding the local-level costs and benefits of ERP through organizational information processing theory», Inform.Manage., vol. 41, $\mathrm{n}^{\circ}$. 4. 431-443, (2004).

10. T. Grossman y J. Walsh, «Avoiding the Pitfalls of Erp System Implementation», Inform.Syst.Manage, vol. 21, n. ${ }^{\circ} 2.38$, (2004)

11. Gartner, «ERP II Vertical Segmentation - Discrete manufacturers beware», Gartner.Inc., 2002.

12. R. Kohavi, N. J. Rothleder, y E. Simoudis, «Emerging trends in business analytics», Commun.ACM, vol. 45, nº. 8. 45-48, (2002).

13. A. Jakupović, M. Pavlic, y M. Asenbrener, «Measuring the Size and Contribution of ERP Solutions through Covered Business Sectors», in ISAS Information Systems.Analysis.Synthesis, Orlando, Florida, (2010).

14. A. Jakupović, M. Pavlic, y P. Poscic, «Business sectors and ERP solutions», in Information Technology Interfaces (ITI), 2010 32nd International, Cavtat/ Dubrovnik. 477-482, (2010).

15. R. Baldrich, M. Vanrell, y J. J. Villanueva, «Polarization and Color Techniques in Industrial Inspection», Munich, Germany, vol. 3826. 124135, (1999).

16. S. Kukkonen, H. Kalviainen, y J. Parkkinen, «Color features for quality control in ceramic tile industry», Opt.Eng., vol. 40, n. 2. 170-177, (2001).

17. F. Alarcón, M. del M. E. Alemany, F. C. Lario, y R. F. Oltra, «La falta de homogenidad del producto (FHP) en las empresas cerámicas y su impacto en la reasignación del inventario», Bol.Soc.Esp.Ceram.Vidrio, vol. 50, nº 1 . 49-58, (2011) 